\title{
A brief history of international meetings of the Western Society of Naturalists
}

\section{Una breve historia de las reuniones internacionales de la Western Society of Naturalists}

\author{
Diana L Steller ${ }^{1}$, Julio Lorda ${ }^{2}$, Rodrigo Beas-Luna ${ }^{2}$, Michael S Foster ${ }^{1}$ \\ 1 Moss Landing Marine Laboratories, San José State University. \\ 2 Universidad Autónoma de Baja California.
}

"The object of this society shall be the stimulation of general interest in the fields of biological science. The society shall be broad enough in scope and liberal enough in its organization to meet the needs of all naturalists."

Western Society of Naturalists By-laws, 1916

Beginning in 1916, the Western Society of Naturalists (WSN) has had an annual meeting goal to be "the provision of opportunities for presentation and discussion of problems of common interest to biologists," and it still attempts to "exercise an integrative function which cannot be performed by more specialized organizations." Since the society's inception, the meeting location has varied annually along the countries and states of western North America, namely the United States, Mexico, and Canada. Varying the meeting location increases attendance opportunities geographically among members. The society has historically encouraged international attendance leading to enhanced research collaborations and student exchanges.

In the United States, meetings have been held in 7 western states, with the majority (82\%) of meeting locations being in California and Washington (Foster et al. 1999). There have been 2 meetings in Mexico and 3 in British Columbia, Canada, and 1 meeting, for the first time, held online in 2020. In 1996 the first Mexican meeting was held in La Paz, Baja California Sur, in collaboration with the Universidad Autónoma de Baja California Sur. It enhanced the cross-border exchange of ideas and both expanded ongoing and initiated new fruitful collaborations. These exchanges continued when the 2019 WSN meeting was held in Ensenada, Baja California, in collaboration with the Universidad Autónoma de Baja California from 31 October to 3 November, 2019. The impressive expansion of Mexican research by Mexican scientists was highlighted in the special plenary session by Hector Reyes Bonilla entitled "Marine research in northwest Mexico: past, present and future" and the wide variety of presentations. The need for ongoing international collaboration in marine research was emphasized in the presidential symposium presentations by Rodrigo Beas-Luna, “Oceans don't have borders, science
"El objetivo de esta sociedad será la estimulación del interés general en las áreas de las ciencias biológicas. La sociedad será suficientemente amplia en su alcance y suficientemente liberal en su organización para satisfacer las necesidades de todo naturalista."

Estatutos de la Western Society of Naturalists, 1916

Comenzando en 1916, la Western Society of Naturalists (WSN) ha tenido como meta para su reunión anual "la provisión de oportunidades para la presentación y discusión de problemas de interés común para biólogos" y todavía intenta "ejercitar una función integrativa que no puede ser llevada a cabo por organizaciones más especializadas." Desde el comienzo de esta sociedad, el lugar de reunión ha variado anualmente a lo largo de los países y estados del oeste de Norteamérica, a saber Estados Unidos, México y Canadá. El variar el lugar de reunión aumenta las oportunidades geográficas de asistencia entre los miembros. La sociedad ha fomentado históricamente la asistencia internacional que lleve a investigaciones colaborativas e intercambios estudiantiles.

En Estados Unidos, se han celebrado reuniones en 7 estados del oeste y la mayoría de ellos ( $82 \%$ ) han sido en California y Washington (Foster et al. 1999). Han habido 2 reuniones en México y 3 en British Columbia, Canadá, y una reunión, por primera vez, celebrada en línea en 2020. En 1996, la primera reunión mexicana se celebró en La Paz, Baja California Sur, en colaboración con la Universidad Autónoma de Baja California Sur. Esta reunión reforzó el intercambio transfronterizo de ideas, además de expandir previas e iniciar nuevas fructíferas colaboraciones. Estos intercambios continuaron cuando el congreso de la WSN en 2019 fue celebrado en Ensenada, Baja California, en colaboración con la Universidad Autónoma de Baja California del 31 octubre al 3 de noviembre de 2019. La impresionante expansión de la investigación en México por científicos mexicanos fue destacada en la sesión plenaria especial por Hector Reyes Bonilla titulada "Marine research in northwest Mexico: past, present and future" y la gran variedad de presentaciones. La necesidad de colaboraciones internacionales continuas en la investigación marina fue enfatizada en las presentaciones del simposio presidencial por Rodrigo Beas-Luna, "Oceans don't have borders, science shouldn't either", y Sergio Navarrete, 
shouldn't either," and Sergio Navarrete, "The importance of cultural diversity for sustainability and the successful development of inter-disciplinary studies." WSN is committed to spatially varying annual meeting locations, including periodically at international venues.

"No doubt the true and most important history of WSN occurs every year but is not recorded - the ideas stimulated and tested, collaborations formed, careers launched, and friendships made and revisited as a result of papers presented and the informal social interactions that follow. The Western Society of Naturalists continues its long tradition of stimulating interest in and understanding nature, and adjusting attitudes" (Foster et al 1999).

International locations of WSN meetings since 1916

1974: University of British Columbia, Vancouver \& Simon Fraser University, Burnaby, British Columbia, Canada (President: G. Frank Gwilliam).

1983: Simon Fraser University, Burnaby, British Columbia, Canada (President: John Pearse).

1996: Universidad Autónoma de Baja California Sur, La Paz, Baja California Sur, Mexico (President: Gregor Cailliet).

2008: University of British Columbia, Vancouver, British Columbia, Canada (President: Kathy Ann Miller).

2019: Universidad Autónoma de Baja California, Ensenada, Baja California, Mexico (President: Brian Tissot).

\section{REFERENCE}

Foster MS, Hansen GI, Amrein YUL. 1999. History of the Western Society of Naturalists. Santa Barbara Museum of Natural History, Contributions in Science, No. 2. Goleta (CA): Wilson Printing. p. 42.
"The importance of cultural diversity for sustainability and the successful development of inter-disciplinary studies". La WSN está comprometida a variar espacialmente el lugar de reunión anual e incluir, periódicamente, localidades internacionales.

"Sin duda, la verdadera y más importante historia de la WSN ocurre cada año, pero no es registrada - la estimulación y pruebas de ideas, las colaboraciones formadas, el inició de carreras y las amistades formadas y reencontradas como resultado de artículos presentados y las interacciones sociales informales que le siguen. La Western Society of Naturalists continúa su larga tradición de estimular el interés en y la comprensión de la naturaleza, y ajustando actitudes" (Foster et al 1999).

Ubicaciones internacionales de los congresos de la WSN desde 1916

1974: University of British Columbia, Vancouver y Simon Fraser University, Burnaby, Columbia Británica, Canadá (Presidente: G. Frank Gwilliam).

1983: Simon Fraser University, Burnaby, British Columbia, Canadá (Presidente: John Pearse).

1996: Universidad Autónoma de Baja California Sur, La Paz, Baja California Sur, México (Presidente: Gregor Cailliet).

2008: University of British Columbia,Vancouver, Columbia Británica, Canadá (Presidenta: Kathy Ann Miller).

2019: Universidad Autónoma de Baja California, Ensenada, Baja California, México (Presidente: Brian Tissot).

Traducido al español por Lucía Rodríguez. 